\title{
Relationship between Markov Chains and Radiation Defect Formation Processes by Ion Irradiation
}

\author{
Anatoly Ivanovich Kupchishin ${ }^{1}$, Evgeniy Vladimirovich Shmygalev ${ }^{2}$, Tatyana Alexandrovna Shmygaleva ${ }^{2}$ \& \\ Almaz Binuruli Jorabayev ${ }^{2}$ \\ ${ }^{1}$ Kazakh National Pedagogical University Abai, Almaty, The Republic of Kazakhstan \\ ${ }^{2}$ Al-Farabi Kazakh National University, Almaty, The Republic of Kazakhstan \\ Correspondence: Almaz Binuruli Jorabayev, Al-Farabi Kazakh National University, Almaty, 050038, The \\ Republic of Kazakhstan. E-mail: jorabayev@inbox.ru
}

Received: October 26, 2014 Accepted: November 6, $2014 \quad$ Online Published: December 14, 2014

doi:10.5539/mas.v9n3p59 URL: http://dx.doi.org/10.5539/mas.v9n3p59

\begin{abstract}
The work was performed within in the context of cascade-probabilistic method, the essence of which is to obtain and further application of cascade-probability functions (CPF) for different particles. CPF make sense probability of that a particle generated at some depth $h^{\prime}$ reaches a certain depth $h$ after the $n$-th number of collisions. We consider the interaction of ions with solids and relationship between radiation defect formation processes and Markov processes and Markov chains. It shows how to get the recurrence relations for the simplest CPF from the Chapman-Kolmogorov equations. In this case the particle does not change its direction of movement after the collision, the flow rate does not depend on time, and consequently, on the depth of penetration. We also obtained recurrence relations for the CPF with the energy loss of ions from the Kolmogorov-Chapman equations, the flow rate depends on the depth of penetration.
\end{abstract}

Keywords: cascade-probabilistic, ion, defect formation, Markov chain, Markov processes

\section{Introduction}

Previously were described the basics of the cascade-probabilistic method and its applications in the physics of cosmic rays, radiation and positron physics. The basics of modelling of radiation defects within the cascade-probabilistic method were obtained. It should be noted that the problems of radiation defect formation in the interaction of ions with matter are presented in a large number of works, for example (Agranovich \& Kirsanov, 1976, Orlova \& Trushina, 1979, Ackerman et al., 1972, Caro, 1993, Zou et al., 1993, Audouard et al., Fink et al., 1993, Konopleva et al., 1971). Energy losses on ionization and excitation of the electron shells of atoms of the environment are not taken into account, so we used a simple CPF.

Since the mileage of interaction $\lambda$ [lambda] for formation of primary knock-on atoms (PKA) is strongly dependent on the energy, it is necessary to obtain the mathematical and physical models of the charged particles through substance. Continuous energy loss of charged particles that occur along the path of their motion in solids is very important. Calculations show that the neglect of the real dependencies of various parameters of the elementary act and others on energy, angle, depth, etc. can lead to significant errors in the calculations.

Based on the analysis and the actual calculation of the cross sections of electron-electron, electron-atom, proton-electron, ion-electron, ion-atom collisions, we can conclude that most of the energy is lost by primary charged particles as they pass through materials for ionization and excitation of the atoms of the environment (the so-called ionization losses). On hundreds of collisions of charged particle with the electrons of the atoms of the solid there are one or two collisions with atoms, nuclei (the same thing happens in the formation of Kumahovskii's radiation in crystals and channelling and so on).

For the above types of processes we propose the following physical model. Charged particle along the path of its motion continuously loses its energy for ionization and excitation (energy loss $d E / d x$ for each class of particles depending on energy are known and are described by analytic expressions, in particular, the Bethe-Bloch). Collisions with atoms, nuclei, or in the channelling and formation of Kumahovskii's radiation occur discretely. After the collision the primary particles retain its direction of motion. When the charged particles move through the substance its mileage depends on the energy of the cross section of interaction. 
It should be noted that previously (Boss and Kupchishin, 1988, Vol.1, p. 112, Boss and Kupchishin, 1988, Vol.2, p. 144) questions of relationship between cascade-probability functions, the spectra's energy of primary knocked-on atoms, the concentration of defects and fluxes of secondary particles $N$, integral multiplicities and others Markov processes were not considered. The study of these relationships has led to expansion of our knowledge of the processes occurring in materials when high-energy particles passes through them and a different look at these phenomena, in particular, from a general standpoint. Actually already obtained analytical expressions for the CPF, the energy spectra of passing and secondary particles $N$ and the defect concentration $C$ and etc. can be derived from the Chapman-Kolmogorov equations, by setting the appropriate physical and mathematical models.

Processes of passing of particles through substance and the formation of radiation-induced defects in it can be viewed as Markov processes, continuous in time and discrete on the number of collisions. The final expression for $\psi, N$, and $C$ are represented as sums, integrals and multiplications of the corresponding conditional probabilities and the normalization coefficients, depending on the type and energy of the particles, the reaction channels, differential and integral cross sections of the interaction, the energy lost from the elemental act, environment density, and etc.

Consider the interaction of charged particles with substance in the generation of radiation defects in solids irradiated by electrons, protons, alpha particles and ions.

It is assumed that the primary particles (electrons, protons, alpha particle or ion) are formed at a depth $h^{\prime}$, is reacted with a substance in following ways:

1. Charged particle loses energy on ionization and excitation (the main type of energy loss). These losses are assumed to be continuous to a depth of the passing of particles.

2. The primary particle forms PKA, and on hundreds of interactions with electrons of the environment (ionization losses) there are approximately a few interactions on the formation of PKA.

3. PKA forms Frenkel pairs (vacancy, interstitial atom) in the case of electron irradiation and cascading regions in the case of proton, alpha and ion irradiation.

4. For electrons the relativistic case is considered, since the kinetic energy of the electrons is comparable or more than the rest energy of the electron interaction cross section is taken as the McKinley-Feshbach or Mott cross sections, ionization losses are calculated from the Bethe-Bloch formula.

5. For protons, alpha particles and ions non-relativistic case is considered, the cross section of the interaction is selected as the Rutherford cross section, ionization losses for protons and alpha particles are calculated from the Bethe-Bloch formula, for ions are taken from the tables of the spatial distribution parameters of ion-implanted impurities (Kumakhov-Komarova).

Consider the system $S$, which is a process of interaction of particles with material and having one, two, three... collisions. Such process is a stochastic process with a discrete number of collisions and continuous in time, and consequently, continuous in depth of particle penetration. Transitions of system $S$ from one state to another occur under the influence of some event streams. Since we consider the ordinary flow of events and without consequences, they are Poisson flow of events. If the events form a Poisson flow, the number of events that occur at any part of the time $\left(t_{0}, t_{0}+\tau\right)$ has a probability distribution law (Guter \& Ovchinskii, 1967, Kolmogorov, 1974, Feller, 1984):

$$
\rho_{n}=\frac{a^{n}}{n !} e^{-a}
$$

where $a$ - the expected number of points falling on the interval:

$$
a=\int_{t_{0}}^{t_{0}+\tau} \lambda(t) d t,
$$

$\lambda(t)-$ flow density or intensity.

If $\lambda(t)=$ const, Poisson process is called stationary Poisson, or just flow.

At a constant flow rate

$$
a=\lambda l \text {. }
$$

Distribution in the form of (1) obtained by eminent French mathematician of the last century, Poisson S.D. 
In our case, the states of the system are related with one neighbour element. This scheme of random process refers to pure breeding scheme, and the process is a pure breeding process. Set of system states is nonergodic, intransitive, not closed, finite, states are irrevocable and non-recurrent, the finite state of the system is absorbing. The process of interaction of particles with material is also a Markov process, since all the probabilities in the future depend only on what state this process is in now and do not depend on how this process took place in the past. A Markov chain is a kind of a Markov process, in which the future depends on the past through the present (Guter \& Ovchinskii, 1967, Kolmogorov, 1974, Feller, 1984, David, 2009, Wentzell, 1996, Norris, 1998).

\section{Method}

The process of interaction of ions with material, including solids, is described as a Markov chain, because the conditional probability of each event in this trial are uniquely determined by the result of the previous state. Markov chain is completely described by specifying of all possible transition probabilities, which are written in the form of a square matrix of order $k$ (Guter and Ovchinskii, 1967, Kolmogorov, 1974, Feller, 1984, Wentzell, 1996).

A Markov chain is a process with discrete states and discrete time, so in order to make the transition from the Markov process with discrete states and continuous time to the Markov chains we need to set a sufficiently small depth interval $\Delta h$, so small that not in one of the Poisson flows acting on the system could not appear more than one event in the depth interval $\Delta h$ (Guter and Ovchinskii, 1967, Kolmogorov, 1974). Let's define for each pair of states $\left(S_{i}, S_{j}\right)$, between which there is a transition $S_{i} \rightarrow S_{j}$, the transition probability $\psi_{i j}(h, \Delta h)=\psi_{i j}(k)$, which corresponds to a penetration depth (Guter and Ovchinskii, 1967, Kolmogorov, 1974, Feller, 1984, Wentzell, 1996). Suppose at some depth $h^{\prime}$ at an angle $\gamma$ [gamma] in the chosen direction (relative to the perpendicular to the sample surface) was generated a particle (nucleon, electron, positron, primary knock-on atom). Let us assume that after the collision, it does not change its direction of movement, the flow rate does not depend on time, and consequently, on the penetration depth, i.e.

$$
\lambda(h)=\lambda=\text { const }
$$

In the future, instead of the time we will consider the depth of penetration. Using the well-known Chapman-Kolmogorov equation for a Markov process, namely (Feller, 1984):

$$
p_{i n}(\tau, t)=\sum_{v} p_{i v}(\tau, s) p_{v n}(s, t)
$$

where $\tau<s<t$, we obtain the recurrence relation for the transition probabilities:

$$
\psi_{i n}\left(h^{\prime}, h, \alpha_{0}\right)=\sum_{v} \psi_{i v}\left(h^{\prime}, h^{\prime \prime}, \alpha_{0}\right) \psi_{v n}\left(h^{\prime \prime}, h, \alpha_{0}\right)
$$

But since the process is continuous on depth of penetration and the particle is always at a certain depth, instead of the sum we have the integral, which is taken over the entire depth of $h^{\prime}$ to $h$. Thus, we obtain the following relations:

$$
\begin{aligned}
& \psi_{n}\left(h^{\prime}, h, \alpha_{0}\right)=\int_{h^{\prime}}^{h} \psi_{k}\left(h^{\prime}, h^{\prime \prime}, \alpha_{0}\right) \frac{d h^{\prime \prime}}{\alpha_{0}} \psi_{n-k-1}\left(h^{\prime \prime}, h, \alpha_{0}\right), \\
& \Psi_{n}\left(h^{\prime}, h, \alpha_{0}\right)=\int_{h^{\prime}}^{h} \Psi_{n-k-1}\left(h^{\prime}, h^{\prime \prime}, \alpha_{0}\right) \frac{d h^{\prime \prime}}{\alpha_{0}} \Psi_{k}\left(h^{\prime \prime}, h, \alpha_{0}\right), k=1 \div(n-1) .
\end{aligned}
$$

Or in a simpler form:

$$
\begin{gathered}
\Psi_{n}\left(h^{\prime}, h, \alpha_{0}\right)=\int_{h^{\prime}}^{h} \Psi_{0}\left(h^{\prime}, h^{\prime \prime}, \alpha_{0}\right) \Psi_{n-1}\left(h^{\prime \prime}, h, \alpha_{0}\right) \frac{d h^{\prime \prime}}{\alpha_{0}}, \\
\psi_{n}\left(h^{\prime}, h, \alpha_{0}\right)=\int_{h^{\prime}}^{h} \psi_{n-1}\left(h^{\prime}, h^{\prime \prime}, \alpha_{0}\right) \psi_{0}\left(h^{\prime \prime}, h, \alpha_{0}\right) \frac{d h^{\prime \prime}}{\alpha_{0}},
\end{gathered}
$$

where $\psi_{n}\left(h^{\prime}, h, \alpha_{0}\right)$ - the probability of $n$ particle collisions, reaching a depth of $h$ - the transition probability in $n$ steps; $\quad \alpha_{0}=\lambda \cos \gamma ; \psi_{n-1}\left(h^{\prime}, h^{\prime \prime}, \alpha_{0}\right)$ - the probability of $(n-1)$ particle collisions - probability of transition in $(n-1)$ step; $\psi_{0}\left(h ", h, \alpha_{0}\right)$ - the probability that a particle reaches the depth $h$, without suffering any collision - probability of transition in 1 step; $\frac{d h^{\prime \prime}}{\alpha_{0}}$ - the probability that the particle is going to collide at a depth $h^{\prime \prime}$. 
From (1) we obtain for $n=0$ the probability that the particle will reach the depth $h$, without suffering any collision:

$$
\psi_{0}\left(h^{\prime}, h, \alpha_{0}\right)=e^{-a}=e^{-\frac{h-h^{\prime}}{\alpha_{0}}}
$$

where $a=\frac{h-h^{\prime}}{\alpha_{0}}$.

Using recursive relation (10), we obtain the probability that the particle will reach a depth $h$, experiencing one, two, ... $n$ collisions in the case where $\lambda$ [lambda] and $\theta$ [theta] are not changed after a collision (Boss and Kupchishin, 1988, Vol.1, p. 112, Boss and Kupchishin, 1988, Vol.2, p. 144, Kupchishin, 1986):

$$
\psi_{n}\left(h^{\prime}, h, \alpha_{0}\right)=\left(\frac{h-h^{\prime}}{\alpha_{0}}\right)^{n} \frac{1}{n !} \exp \left(-\frac{h-h^{\prime}}{\alpha_{0}}\right) .
$$

In this case, the Markov chain is nonuniform because the transition probabilities $\psi_{k}, k=0,1, \ldots n$ [psi $\left.\mathrm{k}_{\mathrm{k}}\right]$ are changed for each step $k$, the flow rate does not depend on the penetration depth, i.e. all flows that transform the system $S$ from one state to another, are the simplest stationary Poisson flows. This Markov chain has not stationary regime, because it does not have the ergodic feature.

The simplest CPF is in the extreme case, namely, when $\frac{h-h^{\prime}}{\alpha_{0}}=\alpha$ goes to the Poisson distribution.

\section{Results}

Consider the case where after the collision particle does not change its direction of movement, the flow rate depends on time, and consequently on the penetration depth, i.e. (Kupchishin et. al, 2010).

$$
\lambda(h)=\frac{1}{\lambda_{0}}\left(\frac{1}{a\left(E_{0}{ }^{\prime}-k h^{\prime \prime}\right)}-1\right) .
$$

But since the process is continuous on depth of penetration and the particle is always at a certain depth, instead of the sum we have the integral, which is taken over the entire depth of $h^{\prime}$ to $h$. Thus, we obtain the following relations (Kupchishin et al., 2010):

$$
\begin{aligned}
& \psi_{n}\left(h^{\prime}, h, E_{0}\right)=\int_{h^{\prime}}^{h} \psi_{k}\left(h^{\prime}, h^{\prime \prime}, E_{0}\right) \psi_{n-k-1}\left(h^{\prime \prime}, h, E_{0}\right) \frac{1}{\lambda_{0}}\left(\frac{1}{a\left(E_{0}^{\prime}-k h^{\prime \prime}\right)}-1\right) \mathrm{dh"}, \\
& \psi_{n}\left(h^{\prime}, h, E_{0}\right)=\int_{h^{\prime}}^{h} \psi_{n-k-1}\left(h^{\prime}, h^{\prime \prime}, E_{0}\right) \psi_{k}\left(h^{\prime \prime}, h, E_{0}\right) \frac{1}{\lambda_{0}}\left(\frac{1}{a\left(E_{0}^{\prime}-k h^{\prime \prime}\right)}-1\right) \mathrm{dh}^{\prime \prime}
\end{aligned}
$$

Or in a simpler form:

$$
\psi_{n}\left(h^{\prime}, h, E_{0}\right)=\int_{h^{\prime}}^{h} \psi_{n-1}\left(h^{\prime}, h^{\prime \prime}, E_{0}\right) \psi_{0}\left(h^{\prime \prime}, h, E_{0}\right) \frac{1}{\lambda_{0}}\left(\frac{1}{a\left(E_{0}^{\prime}-k h^{\prime \prime}\right)}-1\right) d h^{\prime \prime},
$$

where $h^{\prime}, h$ - depth generation and registration of a flying ion; $E_{0}$ - initial ion energy; $\psi_{n}\left(h^{\prime}, h, E_{0}\right)-$ probability that the particle will experience $n$ collisions, reaching a depth $h ; \psi_{n-1}\left(h^{\prime}, h^{\prime \prime}, E_{0}\right)$ - probability that the particle will experience $n$-1 collisions, reaching a depth of $h^{\prime \prime}$ from $h^{\prime} ; \psi_{0}\left(h ", h, E_{0}\right)$ - the probability that the particle will reach a depth $h$, while not experiencing any collision; $\lambda_{0}\left[\operatorname{lambda}_{0}\right], a, E_{0}^{\prime}, k-$ the approximation parameters.

Where we have the expression for CPF ions in the form (Jeleunova et al., 2013): 


$$
\psi_{n}\left(h^{\prime}, h, E_{0}\right)=\frac{1}{n ! \lambda_{0}{ }^{n}}\left(\frac{E_{0}-k h^{\prime}}{E_{0}-k h}\right)^{-l} \exp \left(\frac{h-h^{\prime}}{\lambda_{0}}\right) *\left[\frac{\ln \left(\frac{E_{0}-k h^{\prime}}{E_{0}-k h}\right)}{a k}-\left(h-h^{\prime}\right)\right]^{n},
$$

$n$ - number of interactions.

Consider some properties of cascade-probability functions. CPF should have such properties in a physical and mathematical point of view.

1. CPF domain. Function $\ln \left(\frac{E_{0}-k h^{\prime}}{E_{0}-k h}\right)$ is defined for $\left(h^{\prime}<E_{0} / k ; h<E_{0} / k\right)$, and $\ln \left(\frac{E_{0}-k h^{\prime}}{E_{0}-k h}\right)$ greater than 0 or equal to 0 ; so we have $h>=h$ '; approximation coefficient section must be greater than 0 , i.e., $\sigma(h)>0$, so $\sigma_{0}>0$. $E_{0} / k-1 / a k<h<E_{0} / k$.

Thus, the CRF domain for ions is the interval $\left(E_{0} / k-1 / a k ; E_{0} / k\right)$.

2. $\lim _{h \rightarrow h^{\prime}} \psi_{n}\left(h^{\prime}, h, E_{0}\right)=0 ; \lim _{h \rightarrow h^{\prime}} \psi_{0}\left(h^{\prime}, h, E_{0}\right)=1$.

3. Consider

$$
\lim _{k \rightarrow 0} \psi_{0}\left(h^{\prime}, h, E_{0}\right)=\lim _{k \rightarrow 0}\left(\frac{E_{0}-k h^{\prime}}{E_{0}-k h}\right)^{-l} * \exp \left(\frac{h-h^{\prime}}{\lambda_{0}}\right) .
$$

Making some transformations and limit calculation, we obtain

$$
\lim _{k \rightarrow 0} \psi_{0}\left(h^{\prime}, h, E_{0}\right)=\exp \left(-\frac{h-h^{\prime}}{\lambda_{0}}\right) \text {, где } \frac{1}{\lambda}=\frac{1}{\lambda_{0}}\left(\frac{1}{a E_{0}}-1\right) .
$$

Similarly,

$$
\begin{gathered}
\lim _{k \rightarrow 0} \psi_{n}\left(h^{\prime}, h, E_{0}\right)=\frac{1}{\lambda_{0}{ }^{n}}\left(\frac{E_{0}-k h^{\prime}}{E_{0}-k h}\right)^{-l} \exp \left(\frac{h-h^{\prime}}{\lambda_{0}}\right) * \\
{\left[\frac{\ln ^{n}\left(\frac{E_{0}-k h^{\prime}}{E_{0}-k h}\right)}{n ! a^{n} k^{n}}-\frac{\ln ^{n-1}\left(\frac{E_{0}-k h^{\prime}}{E_{0}-k h}\right)}{(n-1) ! a^{n-1} k^{n-1}}\left(h-h^{\prime}\right)+\frac{\ln ^{n-2}\left(\frac{E_{0}-k h^{\prime}}{E_{0}-k h}\right)}{2 !(n-2) ! a^{n-2} k^{n-2}}\left(h-h^{\prime}\right)^{2}+\ldots+\right.} \\
\left.+\frac{(-1)^{n-1} \ln \left(\frac{E_{0}-k h^{\prime}}{E_{0}-k h}\right)}{(n-1) ! a k}\left(h-h^{\prime}\right)^{n-1}+(-1)^{n} \frac{\left(h-h^{\prime}\right)^{n}}{n !}\right] .
\end{gathered}
$$

After the limit calculation for each member of the sum, we obtain

$$
\lim _{k \rightarrow 0} \psi_{n}\left(h^{\prime}, h, E_{0}\right)=\frac{1}{n !}\left(\frac{h-h^{\prime}}{\lambda}\right)^{n} \exp \left(-\frac{h-h^{\prime}}{\lambda}\right),
$$

i.e. CPF with the energy loss becomes simple CPF.

4. Consider the CPF sum over all possible values of interactions number $n$ : 


$$
K_{\infty}=\sum_{n=0}^{\infty} \psi_{n}\left(h^{\prime}, h, E_{0}\right)
$$

We divide $\psi_{n}$ by $n$ terms:

$$
\begin{aligned}
& \psi_{0}=\left(\frac{E_{0}-k h^{\prime}}{E_{0}-k h}\right)^{-l} * \exp \left(\frac{h-h^{\prime}}{\lambda_{0}}\right) . \\
& \psi_{1}\left(h^{\prime}, h, E_{0}\right)=\frac{1}{\lambda_{0}}\left(\frac{E_{0}-k h^{\prime}}{E_{0}-k h}\right)^{-l} * \exp \left(\frac{h-h^{\prime}}{\lambda_{0}}\right) *\left[\frac{1}{a k} \ln \left(\frac{E_{0}-k h^{\prime}}{E_{0}-k h}\right)-\left(h-h^{\prime}\right)\right] \\
& \psi_{2}\left(h^{\prime}, h, E_{0}\right)=\frac{1}{\lambda_{0}^{2}}\left(\frac{E_{0}-k h^{\prime}}{E_{0}-k h}\right)^{-l} * \exp \left(\frac{h-h^{\prime}}{\lambda_{0}}\right) * \\
& {\left[\frac{\ln ^{2}\left(\frac{E_{0}-k h^{\prime}}{E_{0}-k h}\right)}{2 ! a^{2} k^{2}}-\frac{h-h^{\prime}}{a k} \ln \left(\frac{E_{0}-k h^{\prime}}{E_{0}-k h}\right)+\frac{\left(h-h^{\prime}\right)^{2}}{2 !}\right]} \\
& \psi_{3}\left(h^{\prime}, h, E_{0}\right)=\frac{1}{\lambda_{0}{ }^{3}}\left(\frac{E_{0}-k h^{\prime}}{E_{0}-k h}\right)^{-l} * \exp \left(\frac{h-h^{\prime}}{\lambda_{0}}\right) * \\
& {\left[\frac{\ln ^{3}\left(\frac{E_{0}-k h^{\prime}}{E_{0}-k h}\right)}{3 ! a^{3} k^{3}}-\frac{\ln ^{2}\left(\frac{E_{0}-k h^{\prime}}{E_{0}-k h}\right)}{2 ! a^{2} k^{2}}\left(h-h^{\prime}\right)+\frac{\left(h-h^{\prime}\right)^{2}}{2 ! a k} \ln \left(\frac{E_{0}-k h^{\prime}}{E_{0}-k h}\right)-\frac{\left(h-h^{\prime}\right)^{3}}{3 !}\right]} \\
& \psi_{n}\left(h^{\prime}, h, E_{0}\right)=\frac{1}{\lambda_{0}{ }^{n}}\left(\frac{E_{0}-k h^{\prime}}{E_{0}-k h}\right)^{-l} * \exp \left(\frac{h-h^{\prime}}{\lambda_{0}}\right) * \\
& {\left[\frac{\ln ^{n}\left(\frac{E_{0}-k h^{\prime}}{E_{0}-k h}\right)}{n ! a^{n} k^{n}}-\frac{\ln ^{n-1}\left(\frac{E_{0}-k h^{\prime}}{E_{0}-k h}\right)}{(n-1) ! a^{n-1} k^{n-1}}\left(h-h^{\prime}\right)+\frac{\ln ^{n-2}\left(\frac{E_{0}-k h^{\prime}}{E_{0}-k h}\right)}{2 !(n-2) ! a^{n-2} k^{n-2}}\left(h-h^{\prime}\right)^{2}+\ldots+\right.} \\
& \left.+\frac{(-1)^{n-1} \ln \left(\frac{E_{0}-k h^{\prime}}{E_{0}-k h}\right)}{(n-1) ! a k}\left(h-h^{\prime}\right)^{n-1}+(-1)^{n} \frac{\left(h-h^{\prime}\right)^{n}}{n !}\right] .
\end{aligned}
$$

Group all the members for $\left(h-h^{\prime}\right)^{0},\left(h-h^{\prime}\right)^{1},\left(h-h^{\prime}\right)^{2}, \ldots$

We have

$$
\sum_{n=0}^{\infty} \psi_{0 n}\left(h^{\prime}, h, E_{0}\right)=\exp \left(\frac{h-h^{\prime}}{\lambda_{0}}\right)
$$




$$
\sum_{n=1}^{\infty} \psi_{1 n}\left(h^{\prime}, h, E_{0}\right)=-\frac{1}{\lambda_{0}} \exp \left(\frac{h-h^{\prime}}{\lambda_{0}}\right)\left(h-h^{\prime}\right),
$$

$$
\sum_{n=i}^{\infty} \psi_{i n}\left(h^{\prime}, h, E_{0}\right)=\frac{(-1)^{i}}{i ! \lambda_{0}{ }^{i}} \exp \left(\frac{h-h^{\prime}}{\lambda_{0}}\right)\left(h-h^{\prime}\right)^{i}
$$

Summing over all $i$, we obtain the expression:

$$
K_{\infty}=\lim _{n \rightarrow \infty} \sum_{i=0}^{n} \frac{(-1)^{i}}{i ! \lambda_{0}{ }^{i}} \exp \left(\frac{h-h^{\prime}}{\lambda_{0}}\right)\left(h-h^{\prime}\right)^{i}=\lim _{n \rightarrow \infty} \exp \left(\frac{h-h^{\prime}}{\lambda_{0}}\right) \sum_{i=0}^{n} \frac{(-1)^{i}\left(h-h^{\prime}\right)^{i}}{i ! \lambda_{0}{ }^{i}}=1,
$$

i.e. $K_{\infty}=1$.

5. $\lim _{\lambda_{0 \rightarrow \infty}} \psi_{n}\left(h^{\prime}, h, E_{0}\right)=0 ; \quad \lim _{\lambda_{0} \rightarrow \infty} \psi_{0}\left(h^{\prime}, h, E_{0}\right)=1$.

6. $\lim _{n \rightarrow 0} \psi_{n}\left(h^{\prime}, h, E_{0}\right)=\left(\frac{E_{0}-k h^{\prime}}{E_{0}-k h}\right)^{-l} * \exp \left(\frac{h-h^{\prime}}{\lambda_{0}}\right)=\psi_{0}\left(h^{\prime}, h, E_{0}\right)$.

7. $n \rightarrow \infty$.

Breaking $\psi_{n}\left(h^{\prime}, h, E_{0}\right)$ into $n$ members, calculation the limit of each member and using Stirling's formula, we obtain $\lim _{n \rightarrow \infty} \psi_{n}\left(h^{\prime}, h, E_{0}\right)=0$.

8. $\int_{h^{\prime}}^{h} \frac{\psi_{n}\left(h^{\prime}, h, E_{0}\right) d h}{\lambda(h)}=1$,

where $\lambda(h)=1 /\left(\sigma(h) n_{0}\right)$,

$n_{0}-$ number of target atoms per cubic centimeter.

This integral cannot be expressed in members of elementary functions and, therefore, has been calculated numerically by Gauss's method.

The calculation of CPF results depending on the number of interactions and penetration depth are shown in Tables 1, 2.

Table 1. Dependence of displacement percent of the left and right result area borders on number of interactions for aluminum in aluminum: a) $E_{0}=1000 \mathrm{keV}$; b) $E_{0}=800 \mathrm{keV}$

\begin{tabular}{ccccc}
\hline$h^{*} 10^{5}, \mathrm{~cm}$ & $\mathrm{~B}_{1}, \%$ & $\mathrm{~B}_{2}, \%$ & $N_{n}$ & $\mathrm{~B}_{3}, \%$ \\
\hline 10 & 25 & $-8,67$ & 74 & 16,33 \\
15 & 32,29 & $-21,86$ & 85 & 10,43 \\
20 & 41,52 & $-34,15$ & 100 & 7,37 \\
25 & 53 & $-48,3$ & 130 & 4,7 \\
30 & 67,56 & $-64,8$ & 160 & 2,76 \\
35 & 90,82 & $-90,33$ & 220 & 0,49 \\
\hline \multicolumn{7}{c}{} & $\mathrm{a})$ & & \\
\hline$h^{*} 10^{4}, \mathrm{~cm}$ & $\mathrm{~B}_{1}, \%$ & $\mathrm{~B}_{2}, \%$ & $N_{n}$ & $\mathrm{~B}_{3}, \%$ \\
\hline 1 & 28,8 & 28,6 & 25 & 57,4 \\
5 & 20,46 & 2,63 & 63 & 23,09 \\
9 & 25 & -10 & 78 & 15 \\
13 & 32 & -22 & 90 & 10 \\
17 & 41,10 & $-33,6$ & 105 & 7,5 \\
\hline
\end{tabular}




\begin{tabular}{llccc}
\hline 21 & 51,82 & -47 & 130 & 4,82 \\
25 & 65,93 & $-63,25$ & 160 & 2,68 \\
29 & 87,33 & $-86,6$ & 200 & 0,73 \\
\hline
\end{tabular}

b)

Table 2. Dependence of displacement percent of the left and right result area borders on the depth of penetration for silicon into silicon: a) $E_{0}=1000 \mathrm{keV}$; b) $E_{0}=500 \mathrm{keV}$; c) $E_{0}=100 \mathrm{keV}$

\begin{tabular}{|c|c|c|c|c|c|}
\hline$h^{*} 10^{4}, \mathrm{~cm}$ & $h / \lambda, \mathrm{cm}$ & $\mathrm{C}_{1}, \%$ & $\mathrm{C}_{2}, \%$ & $N_{h}$ & $\mathrm{C}_{3}, \%$ \\
\hline 1 & 933 & 18 & 25 & 20 & 43 \\
\hline 3 & 2918 & 7,9 & 16 & 40 & 23,9 \\
\hline 5 & 5076 & 3,2 & 14,8 & 60 & 18 \\
\hline 7 & 7432 & $-0,5$ & 15,5 & 75 & 15 \\
\hline 9 & 10015 & $-3,6$ & 16,5 & 90 & 12,9 \\
\hline 10 & 11401 & -5 & 16,5 & 110 & 11,5 \\
\hline 30 & 67193 & $-28,08$ & 31 & 500 & 2,92 \\
\hline \multicolumn{6}{|c|}{ a) } \\
\hline$h^{*} 10^{4}, \mathrm{~cm}$ & $h / \lambda, \mathrm{cm}$ & $\mathrm{C}_{1}, \%$ & $\mathrm{C}_{2}, \%$ & $N_{h}$ & $\mathrm{C}_{3}, \%$ \\
\hline 5 & 10758 & $-2,85$ & 14,5 & 100 & 11,65 \\
\hline 10 & 26876 & $-14,65$ & 21,7 & 180 & 7,05 \\
\hline 15 & 54546 & $-24,83$ & 28,5 & 380 & 3,67 \\
\hline 20 & 115467 & $-27,6375$ & 28,8 & 1300 & 1,1625 \\
\hline 25 & 376703 & $-11,9135$ & 11,9182 & 280000 & 0,0047 \\
\hline \multicolumn{6}{|c|}{ b) } \\
\hline$h^{*} 10^{4}, \mathrm{~cm}$ & $h / \lambda, \mathrm{cm}$ & $\mathrm{C}_{1}, \%$ & $\mathrm{C}_{2}, \%$ & $N_{h}$ & $\mathrm{C}_{3}, \%$ \\
\hline 1 & 10018 & $-2,78$ & 15,5 & 90 & 12,72 \\
\hline 1,5 & 16700 & $-8,898$ & 18,5 & 125 & 9,602 \\
\hline 2,0 & 25136 & $-14,8$ & 22,35 & 175 & 7,55 \\
\hline 2,5 & 36204 & $-20,37$ & 26 & 250 & 5,63 \\
\hline 3,0 & 51488 & $-25,1$ & 29 & 375 & 3,9 \\
\hline 3,5 & 74157 & $-27,98$ & 30,15 & 650 & 2,17 \\
\hline 4,0 & 111627 & $-27,145$ & 28,1 & 1500 & 0,955 \\
\hline 4,5 & 186233 & $-20,65$ & 20,832 & 7500 & 0,182 \\
\hline
\end{tabular}

c)

Energy spectrum of primary knock-on atoms (PKA) calculated by the formula (Kupchishin et al., 2010):

$$
W\left(E_{0}, E_{2}, h\right)=\sum_{n=n_{0}}^{n_{1}} \int_{h-k \lambda_{2}}^{h} \psi_{n}\left(h^{\prime}\right) \exp \left(-\frac{h-h^{\prime}}{\lambda_{2}}\right) \frac{w\left(E_{1}, E_{2}, h^{\prime}\right) d h^{\prime}}{\lambda_{1}\left(h^{\prime}\right) \lambda_{2}},
$$

where $\psi_{n}\left(h^{\prime}\right)-\mathrm{CPF}$ in a modified form; $\lambda_{1}, \lambda_{2}$ [lambda1], [lambda2] - mileages on ion - atom and atom atom collisions, correspondingly; $k-$ an integer greater than one; $n_{0}, n_{1}-$ the initial and final value of interactions number in the domain of the CPF; $w\left(E_{1}, E_{2}, h^{\prime}\right)$ - spectrum of PKA in the elementary act; $E_{2}-$ energy of primary knock-on atom.

The expression of the vacancy concentration clusters during ion irradiation of a solid is given by (Kupchishin et al., 2010):

$$
C_{k}\left(E_{0}, h\right)=\int_{E_{c}}^{E_{2 \max }} W\left(E_{0}, E_{2}, h\right) d E_{2}
$$

$E_{2 \max }-$ maximum possible energy, acquired by the atom.

The results of calculations radiation defects concentration are shown in Figure 1 and Table 3. 


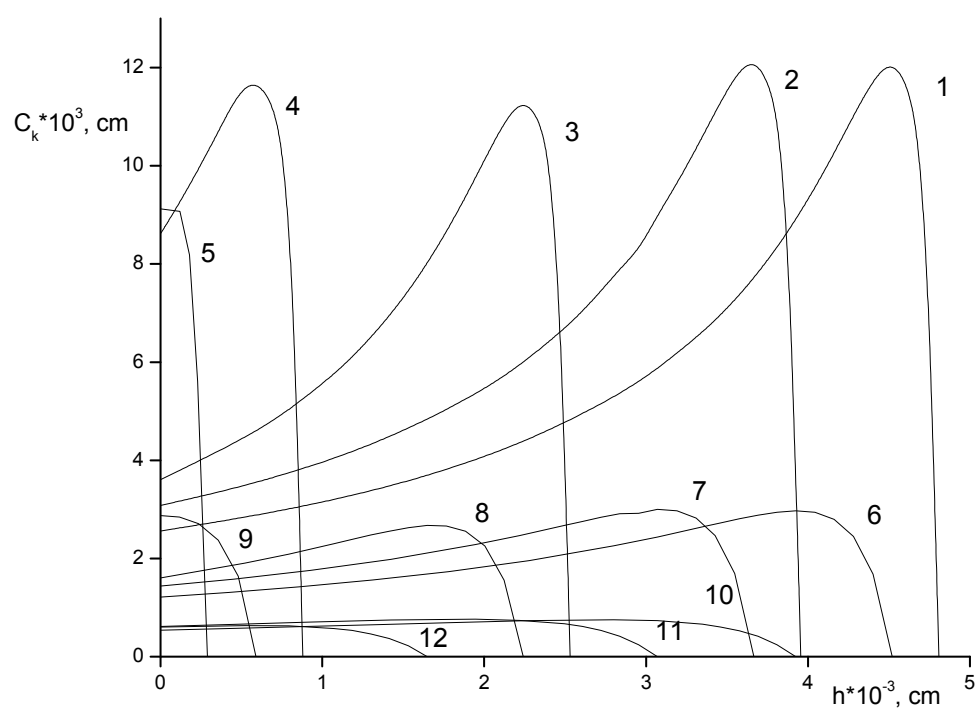

Figure 1. The dependence of the radiation-induced defects concentration on the penetration depth under ion irradiation for silicon in silicon at $E_{c}=50 \mathrm{keV} ; E_{0}=1000,800,500,200,100 \mathrm{keV}(1-5) ; E_{c}=100 \mathrm{keV} ; E_{0}=$ $1000,800,500,200 \mathrm{keV}(6-9) ; E_{c}=200 \mathrm{keV} ; E_{0}=1000,800,500 \mathrm{keV}(10-12)$

Table 3. The borders of the radiation-induced defects concentration domain for nitrogen in aluminum at $E_{c}=50$ $\mathrm{keV}$ and $E_{0}=500 \mathrm{keV}$

\begin{tabular}{ccccc}
\hline$h^{*} 10^{4}, \mathrm{~cm}$ & $C_{K}, \mathrm{~cm}$ & $E_{0}, \mathrm{keV}$ & $n_{0}$ & $n_{l}$ \\
\hline 0,1 & 581 & 500 & 0 & 47 \\
4,5 & 713 & 400 & 345 & 729 \\
6,9 & 800,7 & 350 & 630 & 1122 \\
9,4 & 906 & 300 & 987 & 1585 \\
10,5 & 959 & 280 & 1166 & 1810 \\
11,6 & 1016 & 260 & 1361 & 2050 \\
12,7 & 1076 & 240 & 1573 & 2309 \\
13,8 & 1139 & 220 & 1805 & 2589 \\
14,9 & 1203 & 200 & 2060 & 2893 \\
16,1 & 1279 & 180 & 2368 & 3256 \\
17,4 & 1365 & 160 & 2743 & 3695 \\
18,7 & 1443 & 140 & 3172 & 4191 \\
20 & 1494 & 120 & 3670 & 4762 \\
21,4 & 1488 & 100 & 4306 & 5485 \\
22,8 & 1273 & 80 & 5088 & 6366 \\
23,5 & 975 & 70 & 5555 & 6889 \\
24,3 & 411 & 60 & 6174 & 7579 \\
25 & -738 & 50 & 6815 & 7900 \\
\hline
\end{tabular}

Formula (19) can also be written as an equation of Chapman-Kolmogorov, the flying particle is ion. It is obvious that the interaction of ions with solids, generation of PKA is also described by a Markov chain.

In the expression for the spectrum of PKA (19) there is a multiplication of the probabilities under the integral sign. Here they are: 
1. $\psi_{n}\left(h^{\prime}\right)=\frac{1}{n ! \lambda_{0}^{n}}\left(\frac{E_{0}}{E_{0}-k h^{\prime}}\right)^{\frac{1}{\lambda_{0} a k}} \exp \left(\frac{h^{\prime}}{\lambda_{0}}\right)\left(\frac{\ln \left(\frac{E_{0}}{E_{0}-k h^{\prime}}\right)}{a k}-h^{\prime}\right)^{n}=\psi_{i k}$

$\psi_{i k}\left[\mathrm{psi}_{\mathrm{ik}}\right]$ - the probability that the ion reach a depth $h^{\prime}$ after $(n-1)$-th collision, provided that the previous event occurred, i.e. at a certain depth the primary particle was generated - ion.

2. $w\left(E_{0}, E_{2}, h^{\prime}\right)=\psi_{k m}$ - the conditional probability of forming primary knock-on atom with energy $E_{2}$ from the ion with energy $E_{0}$ after the $n$-th collisions.

3. $\exp \left(-\frac{h-h^{\prime}}{\lambda_{2}}\right) / \lambda_{2}=\psi_{m s}-$ the conditional probability that the PKA, formed at a depth $h^{\prime}$ after the $n$ ion interactions with the substance will reach the depth $\mathrm{h}$.

Spectrum of PKA $W\left(E_{0}, E_{2}, h\right)$ is a probability that from the 1 st ion with energy $E_{0}$ will be generated a certain amount of the secondary particles with energy $E_{2}$ at a depth $h$.

In the general case, all the functions $\psi_{i k}, \psi_{k m}, \psi_{m s}\left[\mathrm{psi}_{\mathrm{ik}}\right]$, [psi $\left.\mathrm{km}_{\mathrm{km}}\right]$, [psi $\left.\mathrm{ms}_{\mathrm{ms}}\right]$ are the transition probabilities of the Markov chain correspondingly from the $i$-th state to the $k$-th, from the $k$-th to the $m$-th, from the $m$-th to the $s$-th.

Then the Chapman-Kolmogorov equation can be written as follows:

$$
\psi_{i j}=\sum_{k} \sum_{m} \Psi_{i k} \cdot \Psi_{k m} \cdot \Psi_{m s},
$$

$\psi_{i j}=W-$ the probability of the transition from the $i$-th state to the $j$-th.

Since the states of the system are continuous in depth, then the expression (22) is transformed into the following:

$$
W\left(E_{0}, E_{2}, h\right)=\psi_{i j}=\sum_{n=0}^{n_{1}} \int_{0}^{h} \psi_{i k} \cdot \psi_{k m} \cdot \psi_{m s} .
$$

\section{Discussion}

Markov chains are used in various areas of scientific research. In chemistry, enzymatic activity, Michaelis-Menten kinetics, can be viewed as a Markov chain, where at each time step the reaction proceeds in a certain direction. While the Michaelis-Menten kinetics is quite simple, much more complex reaction networks can also be modeled by a Markov chain (Kutchukian et al., 2009). In physics, the growth (and composition) copolymers can be obtained using Markov chains. The chain structure can be calculated based on reactivity ratios of the monomers which constitute the growing polymer chain (Kopp et al., 2011). Markov chains are used in finance and economics, to simulate various conditions, including asset prices and the collapse of the market (Prasad et al., 1974, Hamilton, 1989). Our work shows the relationship between the processes of particles interaction with matter and radiation defect formation in solids irradiated by ions with Markov chains and Markov processes. We got recurrence relations for the cascade-probability functions, expressions for the spectra of primary sputtered atoms and the concentration of radiation-induced defects of Markov chains. Similarly, we can show the relationship between the processes of radiation defect formation in solids irradiated by electrons, protons, alpha particles Markov processes and Markov chains.

Similarly, we can show the relationship between the processes of radiation defect formation in solids irradiated by electrons, protons, alpha particles and Markov chains.

Thus, the interaction of particles with solids and the formation of radiation defects in solids irradiated by charged particles can be described by Markov chains and Markov processes.

Unlike others, our researches are used in radiation solid state physics. Also Markov chains and Markov processes can be used in space research. 


\section{Conclusion}

Thus, it is shown that the interaction of particles with material and the formation of radiation defects in solids irradiated by ions is a Markov chain. In this case, the Markov chain is inhomogeneous because the transition probabilities $\psi_{k}, k=0,1, \ldots n\left[\mathrm{psi}_{\mathrm{k}}\right]$ are changed on each step $k$, the flow rate depends on the penetration depth, i.e. all flows that transform system $S$ from one state to another, are non-stationary Poisson flows. Conditional probabilities $\psi_{0}, \psi_{1}, \psi_{2}, \psi_{3} \ldots \psi_{n}\left[\mathrm{psi}_{0}\right],\left[\mathrm{psi}_{1}\right],\left[\mathrm{psi}_{2}\right],\left[\mathrm{psi}_{3}\right] \ldots\left[\mathrm{psi}_{\mathrm{n}}\right]$ are the transition probabilities for inhomogeneous Markov chains with no stationary state. When $k \rightarrow 0$, either $a \rightarrow \infty$, CPF for ions in view of losses of energy goes into the simplest CPF, and therefore goes into the Poisson distribution. The simplest CPF ignores the energy loss on ionization and excitation directly in the process of generating PKA. This Markov chain has not stationary state because they do not have the ergodic property.

In this paper we consider the relationship between the Markov chains and Markov processes to produce models that describe the particles interaction with matter and the formation of radiation-induced defects during ion irradiation.

In the future we plan to use the Markov chain to obtain a cascade-probability functions for unstable particles, muons, pions, neutrons, positrons.

\section{References}

Ackerman, A. F., Nikitushev, Y. M., \& Botwin, V. A. (1972). Using Monte Carlo method in transport problems of fast electrons in matter (p. 162). Alma-Ata: Nauka.

Agranovich, V. M., \& Kirsanov, V. V. (1976). Problems of radiation damage in crystals modeling, 118, 2-51. Moscow, M: UFN. http://dx.doi.org/10.3367/UFNr.0118.197601a.0003

Audouard, A., Balanzar, E., Jousset, J. C., Lesueur, D., \& Thome, L. (1993). Anisotropy of the atomic movements induced in amorphous metallic alloys by swift heavy ion irradiation. Radiation Effects and Defects in Solids (Vol. 126, pp. 93-96). http://dx.doi.org/10.1080/10420159308219686

Boss, E. G., \& Kupchishin, A. I. (1988) Solution of physical problems using cascade- probabilistic method (Vol.1, p. 112). Alma-Ata: Nauka.

Boss, E. G., \& Kupchishin, A. I. (1988). Solution of physical problems using cascade-probabilistic method (Vol.2, p. 144). Alma-Ata: Nauka.

Caro, A. (1993). Molecular dynamic approach to heavy ion impact. Radiation Effects and Defects in Solids (Vol. 126, pp. 15-20). http://dx.doi.org/10.1080/10420159308219671

David, A. L. (2009). Markov chains and mixing times (p. 16). New York, NY: American Mathematical Soc. ISBN 978-0-8218-4739-8.

Feller, W. (1984). An Introduction to Probability Theory and Its Applications (Vol.1, p. 527). Moscow, M: Mir.

Fink, D., Chadderton, L. T., Cruz, S. A., Fahrner, W. R., Hnatowir, V., ... Zaitsev, A. M. (1993). Ion Tracks in condensed carbonaceous matter. Radiation Effects and Defects in Solids (Vol. 126, pp. 247-250). http://dx.doi.org/10.1080/10420159308219719

Guter, R. S., \& Ovchinskii, B. V. (1967). Fundamentals of Probability Theory (p. 159). Moscow, M: Proshveshenie.

Hamilton, J. (1989). A new approach to the economic analysis of nonstationary time series and the business cycle. Econometrica, 57(2), 357-84. http://dx.doi.org/10.2307/1912559

Jeleunova, S. E., Dzhorabaev, A. B., Kupchichin, A. I., \& Shmygaleva, T. A. (2013). Mathematical modelling of radiating processes in iron (pp. 38-42). Almaty, Kazakhstan: Al-Farabi Kazakh National University.

Kolmogorov, A. N. (1974). Fundamental concepts of probability theory (p. 119). Moscow, M: Nauka.

Konopleva, R. F., Litvinov, V. L., \& Ukhin, N. A. (1971). Features of radiation damage to semiconductor high-energy particles (p. 175). Moscow, M: Atomizdat.

Kopp, V. S., Kaganer, V. M., Schwarzkopf, J., Waidick, F., Remmele, T., Kwasniewski, A., \& Schmidbauer, M. (2011). X-ray diffraction from nonperiodic layered structures with correlations: Analytical calculation and experiment on mixed Aurivillius films. Acta Crystallographica Section a Foundations of Crystallography, 68, 148. http://dx.doi.org/10.1107/S0108767311044874 
Kupchishin, A. I. (1986). Interaction of radiation with matter. Cascade-probabilistic method (methodical development for students of the Faculty of Mathematics and Physics) (p. 68). Alma-Ata:Nauka

Kupchishin, A. I., Kylyshkanov, M., \& Shmygaleva, T. A. (2010). Computer simulation and experimental studies of the irradiative processes in iron and hard alloys (p. 263). Almaty, KazNPU named after Abai, KazNU named after Al-Farabi Research Institute.

Kutchukian, P., Lou, D., \& Shakhnovich, E. (2009). FOG: Fragment Optimized Growth Algorithm for the de Novo Generation of Molecules occupying Druglike Chemical. Journal of Chemical Information and Modeling, 49(7), 1630-1642. http://dx.doi.org/10.1021/ci9000458

Norris, J. R. (1998). Markov chains. Cambridge: Cambridge University Press. Retrieved from http://www.statslab.cam.ac.uk/ james/Markov/

Orlova, A. N., \& Trushina, U. V. (1979). Computer simulation of defects in crystals (p. 205). Leningrad, L: FTI named after Ioffe A. N.

Prasad, N. R., Ender, R. C., Reilly, S. T., \& Nesgos, G. (1974). Allocation of resources on a minimized cost basis. 1974 IEEE Conference on Decision and Control including the 13th Symposium on Adaptive Processes, 13, 402-403. http://dx.doi.org/10.1109/CDC.1974.270470

Wentzell, A. D. (1996). Course in the theory of random processes (p. 400). Moscow, M: Nauka.

Zou, Y., Awaya, Y. A., Kambara, T., Kanai, Y., Oura, M., Ando, K., Hitachi, A., \& Kravis, S. (1993). Foil target element and incident energy dependence of multiple inner shell vacancy production of projectile Ar ions. Radiation Effects and Defects in Solids, 126, 87-90s. http://dx.doi.org/10.1080/10420159308219685

\section{Copyrights}

Copyright for this article is retained by the author(s), with first publication rights granted to the journal.

This is an open-access article distributed under the terms and conditions of the Creative Commons Attribution license (http://creativecommons.org/licenses/by/3.0/). 